\title{
Managing Process Assets in a Global IT Service Delivery Environment
}

\author{
Melissa Buco ${ }^{1}$, Hani Jamjoom ${ }^{1}$, Tom Parsons ${ }^{2}$, and Scott Schorno ${ }^{3}$ \\ 1 IBM Research, Hawthorne, NY, USA \\ 2 IBM GTS, Research Triangle Park, NC, USA \\ 3 IBM GTS, Fishkill, NY, USA
}

\begin{abstract}
At IBM, we recognize that our processes are our business. This is especially true in the area of IT Delivery where we have long been focused on the management and reuse of process assets. The current economic climate and advances in technology are rapidly driving IT Delivery to a truly global model. This transition greatly expands the scope of the process assets which need to be managed at a global level to include even the lowest level processes for service delivery. Customers, many of whom are also global, expect consistent quality and reasonable cost, regardless of from where services are delivered. The global management and reuse of IT Delivery process assets at all levels is no longer a desired objective but rather a business imperative. In this paper, we describe a system we are developing to manage, govern, and evolve process assets on a global scale by leveraging expertise of the entire IT Delivery community. We describe the history of the effort, the business drivers, the challenges and solutions we have devised, as well as future work.
\end{abstract}

Keywords: crowd computing, IT service delivery, business process management, governance, service quality.

\section{Introduction}

There are any number of industry standards, such as, ITIL [2] and ISO9000 [1], which apply to IT Service Delivery. It is the responsibility of an organization's management to define the high level processes in conformance with the appropriate industry standards. Although these processes are essential for the overall operation, the vast majority of the daily work performed by an IT service delivery organization happens at much lower levels. For example, the Change Management process governs much of what is done by the delivery personnel. However, the average system administrator is more concerned with the procedure for provisioning a new database server or work instructions for executing specific tasks needed to fix a particular problem or for installing a particular product on a given operating system (see Fig 1). The extremely large number and dynamicity of services offered, the diversity of customers, the heterogeneity of the customers' IT environments, and the global distribution of delivery personnel make managing these process assets and driving standardization to 
best practices a challenging problem of considerable scope. Moreover, the need for some level of customization to address customer specific business needs must also be taken into account.



Fig. 1. Process levels

History and Related Work. At IBM, the enterprise level IT Service Delivery processes have been managed and continually improved for many years at a global level. However, lower level process assets have historically been created in a variety of formats and types of repositories by each delivery location. This has made standardization which is essential for driving down cost challenging.

Several years ago a group in IBM Research began a work on a system called Cyano [7] as part of a broader effort to capture, socialize, and evolve lean [3] best practice procedures executed by the IT delivery teams and links these procedures to the enterprise level processes. The resulting system captured approximately 600 procedures with a global user community of 13,000 users and more than 65,000 user annotations such as process variations and suggestions for improvement. Cyano was a Web based application that enabled the capture, display, and annotation of best practice assets. These assets were shown as flowchart diagrams together with a text description. Cyano provided a single interface into global process components such as: enterprise processes, procedures, tools, and variances. Cyano was limited to supporting process and procedures assets only.

Within the past year, Cyano has evolved into a business process management system (bpms)and been established as the strategic repository for process assets in IT Delivery. In bpms, there is an increased focus on content quality, standardization, and governance. The scope of the effort has also been expanded to address the lowest level process assets, that is, work instructions and other supporting documents which can be customer specific. It is at this low level where the bulk of process assets exist, where most of the delivery team operates, and therefore where the greatest impact of standardization and reuse can be achieved.

The lowest level process assets, such as, instructions for performing specific tasks have long been captured in various local repositories in various formats and levels of detail by teams delivering services to specific accounts. Efforts to 
standardize across teams for areas of interest, e.g. Unix and Intel, have had limited success. This is largely due to the effort required to develop standards across many groups.

Business process management is a broad area in enterprise operation and management. A number of commercial systems have been used heavily for various purpose, such as IBM MQSeries for workflow and message management, SAP or Oracle for process and data management. Many research efforts have been focusing on framework design and process integration, transformation, and verification. Examples of such research include process transformation and integration [6]5], and verification framework for web service [9].

One work on this area is by Yang et al. 10] in which they propose a social network framework for Web 2.0 application. Our work has a different focus, namely, on leveraging social network and data mining for IT Delivery processes.

Benefits. As a global repository for processes, bpms is an essential component in eliminating variability and driving standardization of processes. Less variability equates to improved reliability, service level attainment, and reduced costs of service delivery. The use of consistently documented processes in bpms is also expected to reduce human error. Moreover, bpms will facilitate training which is important in an industry with a large number of sub-contracted resources and a high rate of turnover.

Having assets in a common repository with an assessment and review process will facilitate the identification of best practices and reduce the total number of processes needed. In some cases, bpms will also enable the identification of "gaps" or deficiencies in existing processes. Additionally, bpms will be useful in transforming to best practices during the on boarding new customers.

The remainder of this paper is organized as follows: in Section 2 we discuss the design of bpms, in particular, the role of crowd computing and the importance of governance,linking of process assets, and continual improvement; in Section 3 we discuss future work; in Section 4 we summarize this work in progress.

\section{Bpms Design}

Bpms and its predecessor Cyano were designed to be both scalable and extensible solutions for managing process assets. Some of the key design points were the involvement of the community by using crowd computing technologies, the need for governance to maintain consistency and quality, the ability to link and categorize assets to facilitate navigation and profile user interests, and the importance of continual improvement to maintain the vitality of the content.

Crowd Computing. When we began work on Cyano, it was evident that community involvement was a critical success factor to be able to capture and evolve assets at the procedure level. As we move to a global delivery organization, the value of standardization at the work instruction level in term of cost and quality becomes increasingly clear. The size of this effort is considerable - two to three 
orders of magnitude greater that the Cyano effort. Therefore, the need for community participation is absolutely essential. Certainly, management support and incentives for community participation will be required.

The adoption of social computing technologies by enterprises has not been very far reaching to date. For the Cyano project, it was an ideal fit. The ability to leverage the wisdom of the crowd, that is, the expertise of the subject matter experts across the delivery organization, was exactly what was needed in order to capture the best practice procedure being used in the field. In addition to providing a global environment in which users could record process assets, Cyano also provided a means of registering usage and any process variations, suggesting improvements, and commenting on usability. The expanded scope of bpms has increased its audience and heightened the usefulness of and necessity for community involvement.

Governance. In most social computing applications, governance is not a major concern. However, with bpms, we had an overriding need for standardization, high quality, and consistency. Our goal has been to involve the community while making sure at the same time to incorporate their input in a consistent and controlled manner in order to enhance the quality and usefulness of the community's input.

One community involvement technique is to use folksonomy 4]. A folksonomy is a social networking tagging or indexing classification system derived from collaboration which allows users to suggest new category values as a way to improve the users ability to navigate the system. We chose to allow bpms users to propose new categories which are reviewed prior to becoming visible to the community in order to ensure the validity and usefulness of the category. In this way we can eliminate having different spellings and abbreviations for the the same category.

The bpms approach is to encourage community participation while controlling the overall quality of the assets stored in the system. To achieve this, each asset in bpms has a designated owner or group of owners. The owner is encouraged to leverage user feedback on the asset for the purpose of continual asset improvement. Users enrich the asset through the use of annotations. Annotations can be applied against the asset as a whole or just parts of the asset. In bpms, an annotation is the adding of commentary or explanatory notes, such as references, tools, and variations, to a process artifact. An annotation can be entered for a specific account or a group of accounts (known in bpms as an Account Group). Examples of bpms annotations include: Overview, Policy, Reference (includes URL), Tool, and Variation. All general users can author annotations, but general users can not edit or delete the annotations of other users. A general user is allowed to edit or delete annotations he or she authors.

The editing and deleting of other users annotations is restricted to a set of bpms power users. In IT Delivery, the responsibility of "Power User" is assigned to process leaders responsible for their bpms content. Power Users can also link and unlink assets to other assets or categories. General users are not allowed to manage asset links. 
Bpms assets can be created using business modeling tools such as WebSphere Business Modeler (WBM) and Rational Method Composer (RMC). Assets can also be created and stored in bpms as attachments to a bpms web form using tools such as Microsoft Word, Power Point, and Visio. Assets can also be created directly using a Rich Text style editor built into a Web form. This powerful bpms capability allow assets to be created using a variety of authoring tools which minimizes concerns regarding asset migration to bpms. General users are allowed to author work instructions, while only power users are allowed to author procedures or processes in bpms.

Linking of Assets. Our experience with Cyano has shown that the ability of our users to locate the process assets readily is crucial. Toward achieving that end, we have found the linking the process assets to one another and to a variety of hierarchical categories greatly enhances the users' ability to define their scope of interest and locate assets of interest. For example, users link process assets to the owning organization, geography and accounts where used, delivery catalog entry for which applied, etc. The user can then specify his/her interests in terms of the same categories to scope the set of process assets to be shown. Bpms has the ability to easily define new categories and even content types to meet the needs of the community. In addition, process assets are linked to reflect the natural hierarchy of work instructions supporting procedures or other work instructions, procedures linking to processes or other procedures.

Continuous Improvement. Continuous improvement is a well recognized requirement in the business of IT Delivery. We understand that the usefulness of bpms to the delivery community is directly related to the vitality and relevance of the process assets it contains. Feedback of the usefulness of the assets, suggestions for improvement, ratings, usage counts, registering process variations, and linkage between related processes can all be used by asset owners to evolve the assets and drive standardization. Our goal is to record all process assets in use by the organization and continually update these and maintain the minimal set needed to support the business.

\section{Future Work}

There are many areas which we plan to explore in our future work on the bpms project. In this section, we will address a subset of areas which we considered particularly important.

Incenting Participation. A system such as bpms relies on community participation. How to incent users to participate is an ongoing issue but especially at start up. In the case of the Cyano, users were mandated to record their procedures in Cyano. Simple incentive such as a top contributors' list have proved popular. Clearly, the value of the system and of participation by community members has to be recognized and encouraged by management for the system to succeed. 
Linking Process Assets to the Execution Environment(s). An eventual goal of bpms is to link process assets to the environment(s) in which these assets are executed. For example, if a user is assigned a task in a particular execution tool, we plan to present the user with the appropriate set of process assets (e.g. procedures, work instructionm and other supporting documents) needed to complete the task. This capability relies on having a well categorized process assets and an API for obtaining assets from the bpms system

Tooling to Help Manage Feedback. As the size of the bpms repository grows, there is potential for an asset owner to receive more feedback that can easily be handled. To address this situation, we are exploring tooling which will assist the owner with identifying those ideas which are common and have reached a level of maturity to warrant action.

\section{Conclusion}

Managing a large scale process asset repository for a large community of users presents a number of challenges in terms of usability, governance, and vitality. However, we believe for a large scale global IT Delivery organization such a repository is a necessity. The effort to create and maintain the repository is considerable but the return in terms of reduced cost and increased quality is also considerable and a critical success factor in today's economy.

\section{References}

1. http://www.iso.org/iso/home.htm

2. http://www.itil-officialsite.com/home/home.asp

3. http://www.lean.org/whatslean/

4. http://www. useyourweb.com/blog/?p=62

5. Chituc, C.-M., Toscano, C., Azevedo, A.L.: Collaborative business processes integration and management - lessons learned from industry. In: IEEE SCC, pp. 451-457 (2007)

6. Fujiwara, K., Ramachandran, B., Koide, A., Benayon, J.: Business process transformation wizard: a bridge between business analysts and business process transformation technology. In: IEEE SCC, pp. 83-90 (2007)

7. Jamjoom, H., Qu, H., Buco, M.J., Hernandez, M., Saha, D., Naghshineh, M.: Crowdsourcing and service delivery. IBM Systems Journal (2009)

8. Luo, N., Yan, J., Liu, M.: Towards efficient verification for process composition of semantic web services. In: IEEE SCC, pp. 220-227 (2007)

9. Moser, S., Martens, A., Gorlach, K., Amme, W., Godlinski, A.: Advanced verification of distributed ws-bpel business processes incorporating cssa-based data flow analysis. In: IEEE SCC, pp. 98-105 (2007)

10. Yang, S.J.H., Zhang, J., Chen, I.Y.L.: Web 2.0 services for identifying communities of practice through social networks. In: IEEE SCC, pp. 130-137 (2007) 